\title{
Assessing routine health information system in selected PAIMAN districts by using lot quality assurance sampling (LQAS) technique
}

Pakistan Initiative for Mothers and Newborns (PAIMAN)

Follow this and additional works at: https://knowledgecommons.popcouncil.org/departments_sbsr-rh

Part of the Demography, Population, and Ecology Commons, Family, Life Course, and Society Commons, Gender and Sexuality Commons, International Public Health Commons, Maternal and Child Health Commons, Medicine and Health Commons, and the Women's Health Commons How does access to this work benefit you? Let us know!

\section{Recommended Citation}

Pakistan Initiative for Mothers and Newborns (PAIMAN). 2008. "Assessing routine health information system in selected PAIMAN districts by using lot quality assurance sampling (LQAS) technique." Islamabad: Population Council. 


\section{Assessing Routine Health Information System in Selected PAIMAN Districts by using Lot Quality Assurance Sampling (LQAS) Technique}





\section{Assessing Routine Health Information System in Selected PAIMAN Districts by using Lot Quality Assurance Sampling (LQAS) Technique}




\section{(P) Population Council}

The Population Council, an international, non-profit, non-governmental organization established in 1952, seeks to improve the well-being and reproductive health of current and future generations around the world and to help achieve a humane, equitable, and sustainable balance between people and resources.

The Council analyzes population issues and trends; conducts research in the reproductive sciences; develops new contraceptives; works with public and private agencies to improve the quality and outreach of family planning and reproductive health services; helps governments design and implement effective population policies; communicates the results of research in the population field to diverse audiences; and helps strengthen professional resources in developing countries through collaborative research and programs, technical exchanges, awards, and fellowships.

\section{For inquiries, please contact:}

Population Council

\#7, Street 62, F-6/3, Islamabad, Pakistan

Tel: 92512277439

Fax: 92512821401

Email: info@pcpak.org

web: http://www.popcouncil.org

Layout \& Design : Ali Ammad

Printed by: Crystal Printers, Islamabad

\section{Disclaimer:}

"This study/report is made possible by the generous support of the American people through the United States Agency for International Development (USAID). The contents are the responsibility of JSI Research \& Training Institute, Inc. and do not necessarily reflect the views of USAID or the United States Government." 


\section{Contents}

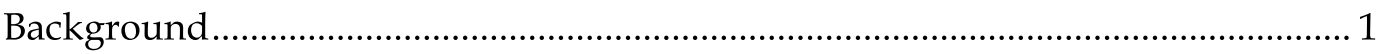

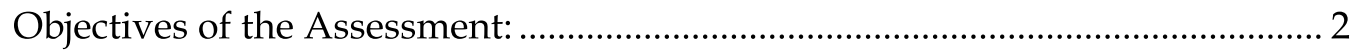

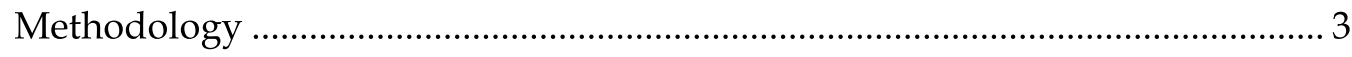

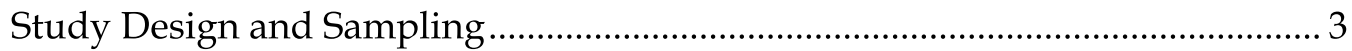

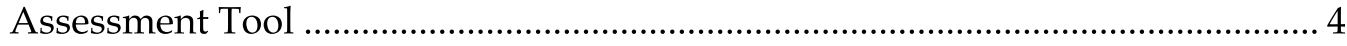

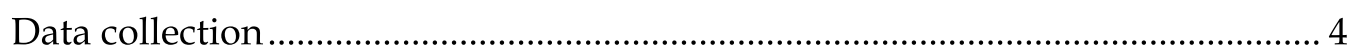

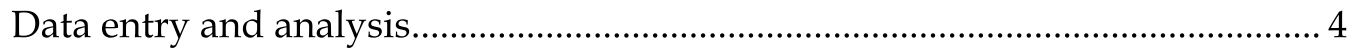

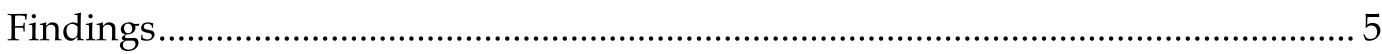

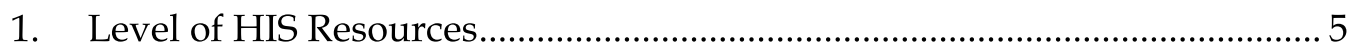

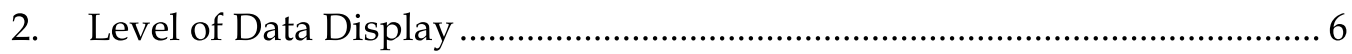

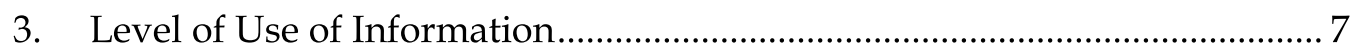

4. Level of Feedback from District /Tehsil Office ........................................... 8

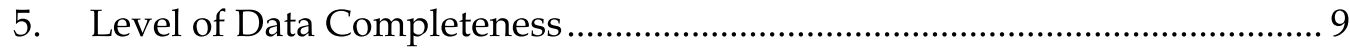

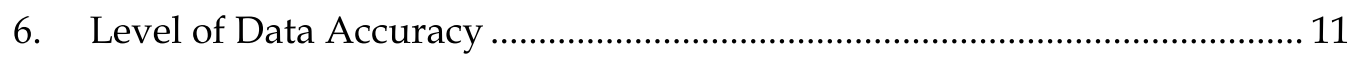

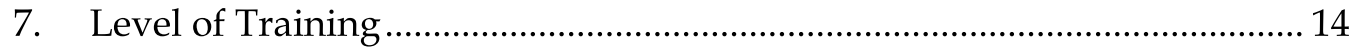

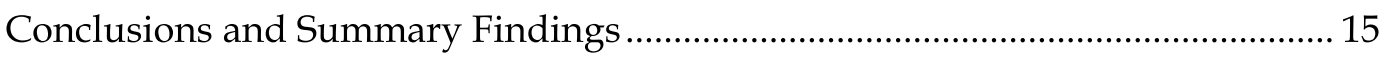

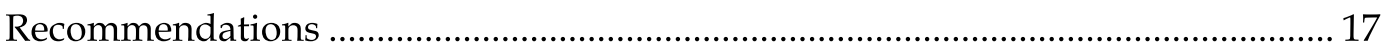

1. Recommendations for improving data completeness and accuracy:.........17

2. Recommendations for improving use of information generated by

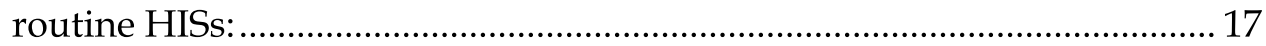

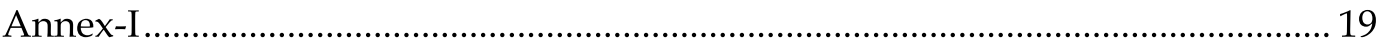

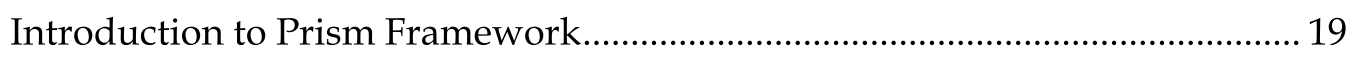

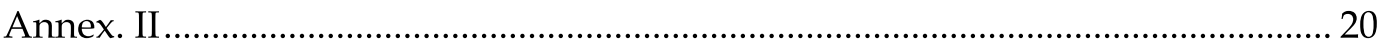

HMIS Record Keeping Registers / Chart........................................................... 20 



\section{Background}

The Pakistan Initiative for Mothers and Newborns (PAIMAN) is a five-year project funded by the United States Agency for International Development (USAID). Its goal is to reduce maternal and newborn mortality in Pakistan, through viable and demonstrable initiatives and capacity building of existing programs and structures within health systems and communities to ensure improvements and supportive linkages in the continuum of health care for women from home to hospital.

Health system strengthening and capacity building of district health managers are important components of the PAIMAN project. The M\&E plan envisages building capabilities of the health system at district level to institutionalize monitoring and evaluation tools and best practices. The aim of capacity building in $\mathrm{M} \& \mathrm{E}$ is to enhance the capabilities of the district health officials on:

- Evidence-based decision making

- Improving quality and utilization of Health Information Systems (HISs)

- Designing, implementing simple strategies using LQAS and related methods to obtain supplemental information for monitoring purposes

PAIMAN with its focus on maternal and neonatal health (MNH) is compiling data relevant to MNH from three available data sources viz. HMIS, LHW-MIS and EPI-MIS. The project is making efforts for improving the functioning of these information systems for efficient management of health services at the district level through generation of quality data and their continuous use for evidence based decision making. Assessment of these HISs was carried out by the project to understand the current quality of data and use of information generated by these information systems so that weaknesses could be identified and appropriate technical assistance can be given to the districts to improve the functioning for the benefit of the project as well as the district health systems at large.

Lot Quality Assurance Sampling (LQAS) technique is simple in its nature and can be used with relatively modest resources. A sample of 19 is used for the assessment of selected performance indicators. It therefore does not need a strong background knowledge of statistics for its learning and application. PAIMAN has introduced this technique for strengthening the monitoring \& evaluation capacity of district health officers and managers and has provided 
them with both theoretical/classroom training and field training in the Project districts.

The LQAS technique was implemented in five districts: Rawalpindi, Sukkur, Jhelum, Lasbella and D. G. Khan, for two assessment studies, viz. i) assessing the performance of HMIS, LHW-MIS and EPI-MIS using the PRISM Performance Diagnostic tool, and ii) assessing sub-grantee NGOs' activities to improve awareness on maternal \& newborn health (MNH) in selected union councils of the Project districts.

This report depicts the Health Information Systems (HISs) assessment applying the LQAS technique and using the PRISM approach which measures the performance of routine health information system and analyzes the technical, behavioral and organizational determinant of that performance. (An introduction to Prism is provided in Annex-I). The Population Council conducted the assessment. Other key partners also involved in this activity at various implementation phases were JSI, Contech International and district health managers.

\section{Objectives of the Assessment:}

The goal of this assessment was to strengthen the routine HISs in the districts so that they can contribute to the improvement of the district health system by providing reliable evidence for management decision making. Further, since improvement of HIS is a continuous process, it is also necessary that the districts should be able to periodically improve the HISs through applying a problem solving approach.

Against this backdrop, PRISM tools were applied in 5 districts using LQAS technique with the objectives to:

1. Build the capacity of the district managers to carry out regular monitoring of the routine HIS performance in their own districts using LQAS technique

2. Assess the performance of routine HISs in the Project districts

The primary foci of the HISs assessment were completeness and accuracy of the data and the level of use of information generated through the routine HISs at the facility level. One commonly mentioned reason of low performance of HIS in Pakistan is the lack of HIS supplies; thus, assessment of the level of HIS resources was also part of the exercise. 


\section{Methodology}

We assessed three main health information systems (HISs) in the public health sector, viz. Health Management Information System for First Level Care Facilities (HMIS-FLCF), Lady Health Workers Program Management Information System (LHW-MIS) and Expanded Program for Immunization Management Information System (EPI-MIS). These three routine health information systems are almost universally implemented in all the primary level (or first level care) health facilities and provide the main bulk of information in the public health sector.

Before conducting this exercise, all EDOs (Health) were informed in writing followed by telephonic briefing on the assessment to be undertaken in their districts. District health managers were identified with the help of EDO $(\mathrm{H})$ to participate in the practical learning of LQAS technique through the assessment of HISs.

In each district, four teams of selected district health managers along with Population Council/JSI/Conetch members visited and assessed quality, completeness, and use of HISs data at the facility level. On the first day, the district managers were given orientation on the LQAS technique which included complete listing of health facilities in the district, sampling procedure, selection of the sample and understanding the questionnaire. The next 2 to 3 days were spent in fieldwork to collect data from health facilities. On the final day, data were tabulated and results disseminated to the $\mathrm{EDOH}$ and district health managers.

\section{Study Design and Sampling}

The HIS assessment was a cross-sectional study that was implemented in the districts of Rawalpindi, Sukkur, Jhelum, Lasbella and D.G. Khan during the months of February- August, 2007. Lot Quality Assurance Sampling (LQAS) technique randomly selected 19 first level care health facilities (17 Basic Health Units and 2 Rural Health Centers) in each district. The selection of the health facilities (BHUs and RHCs) was made randomly from the list of health facilities using random numbers. 


\section{Assessment Tool}

A modified shorter version of PRISM Performance Diagnostic Tool adapted to Pakistani context and for assessing the HMIS-FLCF, LHW-MIS and EPI-MIS, was used. Questions on the training of the HISs were also included to understand the process of the training that was conducted by the Project earlier in 2005. The assessment was first initiated in Rawalpindi district, and based on the experience, the tools were further improved.

The PRISM Performance Diagnostic Tool was used for assessing the levels of HIS resources, accuracy of data transferring from records to reports, completeness of the reports, display of data and use of information in the health facilities. Initially, researchers assessed accuracy and completeness of two monthly reports using a single randomly selected data item from each report (District Rawalpindi). The tools were later amended to assess data accuracy and completeness of only one month (previous month's) report using two data items selected randomly each from the HMIS-FLCF, the LHW-MIS and the EPI-MIS. The selection of data items for assessment was made before the implementation of the assessment and was uniformly applied to all the facilities. Observations on feedback to the health facilities from the district were also included in the amended assessment tool.

\section{Data collection}

Each team comprising a district manager and one of the PAIMAN partners was assigned five health facilities. A field data collection plan with routes was prepared to implement the exercise. In the case of LHW's records, one LHW was randomly selected from the list of LHWs attached to the selected health facility. The Health House of that LHW was visited to cross-check data from her records and her monthly report. Where this was not feasible, the LHW was invited to the health facility to come along with her records.

\section{Data entry and analysis}

Data from the assessment checklist (PRISM Performance Diagnostic Tool) was transferred into an Excel file. Data entry and analysis was done on-site, the analyzed data was then shared and discussed with district health managers and areas of improvement were identified. The total number of positive answers for each question was compared with the LQAS table to determine the level of data accuracy \& completeness, display of data and information use and level of HIS resources. 


\section{Findings}

\section{Level of HIS Resources}

The HMIS-FLCF comprises 19 record-keeping registers/chart (details are given in Annex. II). Monthly facility reports are prepared using data from most of these registers, particularly the OPD Register and the Maternal Health Register. LHWs have their own record-keeping and reporting system. Daily EPI Register and EPI Permanent Register were maintained by the vaccination staff for EPI. Monthly reports are prepared from data recorded in these registers. Availability of these printed HIS tools is essential for proper functioning of the respective HIS. Irregular supplies of printed instruments / tools has affected regular data collection and reporting. Levels of HIS resources were assessed in project districts by examining the availability of following registers/reports in the health facilities.

1. OPD Register

2. Maternal Health Register

3. HMIS-FLCF Monthly Report Forms

4. LHW Monthly Facility Report Forms

5. EPI Daily Register

6. EPI Monthly Report Forms

Figure-1 below shows that availability of HIS resources is satisfactory except in Lasbella. The level of HIS resource availability at the health facilities in Rawalpindi district was 90 percent followed by Sukkur (85 percent), Jhelum (80 percent) and DG Khan (75 percent). Lasbella lagged behind; there the level of HIS resource availability was only 55 percent. The weighted average for all districts regarding the level of availability of HIS resources was 69 percent. 
Figure 1: Availability of HIS Resources at Health Facilities

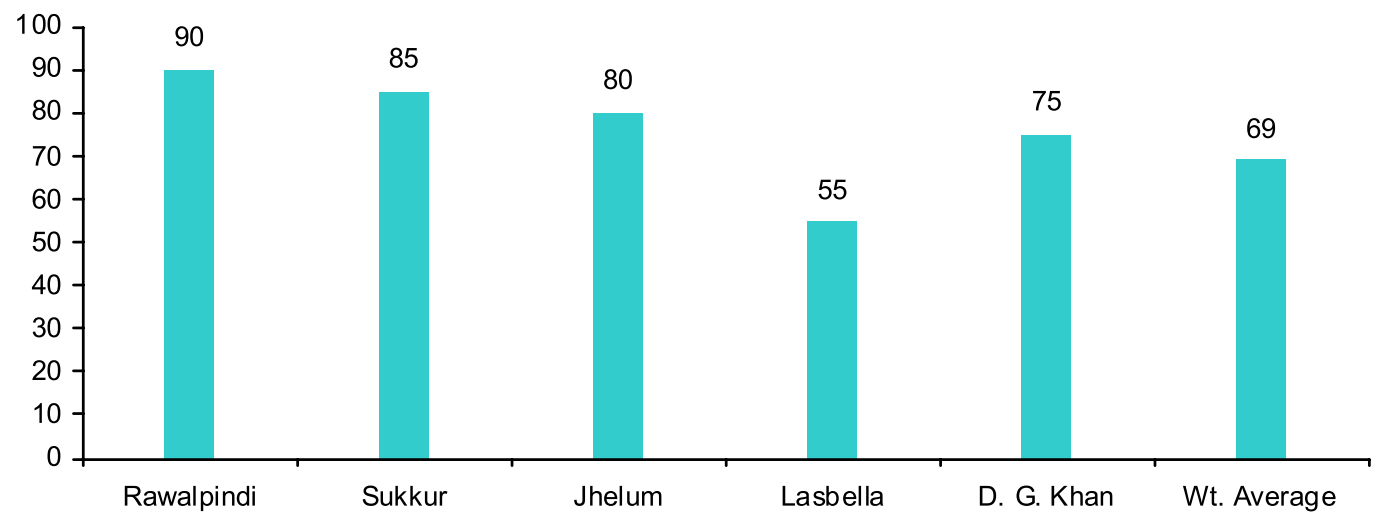

\section{Level of Data Display}

Display of data provides an immediate snap-shot of the situation to the facility in-charges and supervisors and increases accountability. Both numerical and graphical format display formats have an importance. It is a step towards use of data for evidence-based decision making. In fact, there are instructions on graphic display of trends of key indicators in HMIS instructional manual of Government of Pakistan, 2000 (Revised Edition). Therefore, the display of data in the form of numbers or graphs on the walls of the facility was also assessed. This included number/percentage coverage of fully immunized children of less than one year, antenatal care visits, number of deliveries conducted by skilled persons, postnatal care visits and number of OPD patients visiting the facility.

Figure-2 shows the proportion of health facilities displaying the HIS data on the walls in the project districts. In general, data at the majority of health facilities of Jhelum and Rawalpindi were displayed on printed wall charts However, there is a lot of variation: in DG Khan, the level of data display was 35 percent; in Sukkur, the display of EPI data was widespread, but the display of two or more indicators, especially from other routine reports was about 25 percent. In Jhelum, data display was almost universal. There was no display of data at health facilities of Lasbella district. The weighted average for five districts with respect to data display at health facilities was $35 \%$. 
Figure 2: Health Facilities by Level of Data Display

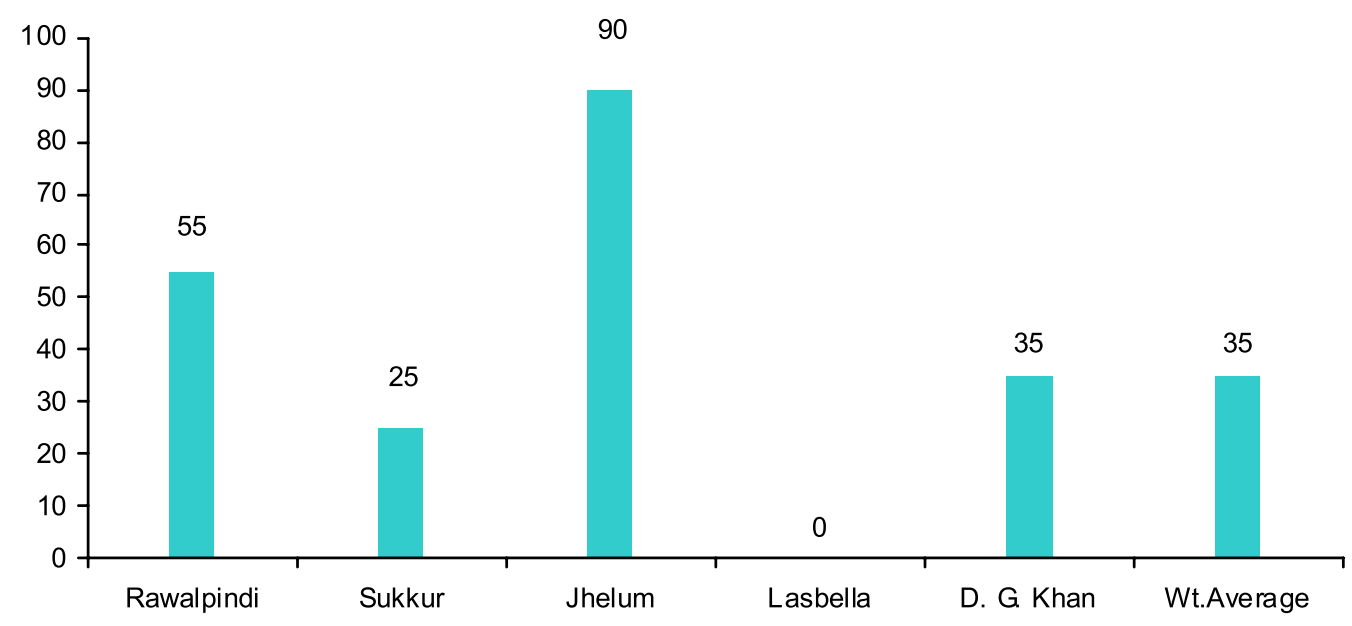

\section{Level of Use of Information}

Even when data are generated by various Health Information Systems, it is of no use if it is not properly understood, utilized and disseminated. As a rule, facilities are required to hold monthly staff meetings to review their performance, discuss problems and to seek their resolutions. Every health facility is supposed to maintain a Meeting Register. The purpose of this register is to keep record of meetings, trainings or field visits held at or organized by the first level care facilities.

One of the indicators of this assessment was to evaluate whether health facilities were holding monthly staff meetings where data/information from the HISs is used for performance improvement. Also, the level of emphasis given to data quality improvement during these meetings was assessed. Availability of written records, especially meeting minutes were taken as evidence of holding facility staff meetings. These documents were then further examined for records on decisions taken utilizing HIS data and discussions on data quality.

Figure-3 shows that the level of use of information and emphasis on data quality among the health facilities is low; in none of the districts did it surpass the 50 percent level. Sukkur, Jhelum and D.G.Khan are at the same level. On average, the level of use of information and emphasis on data quality was 11 percent and 15 percent, respectively. 
Figure 3: Degree of Use of Information and Emphasis on Data Quality in Health Facilities

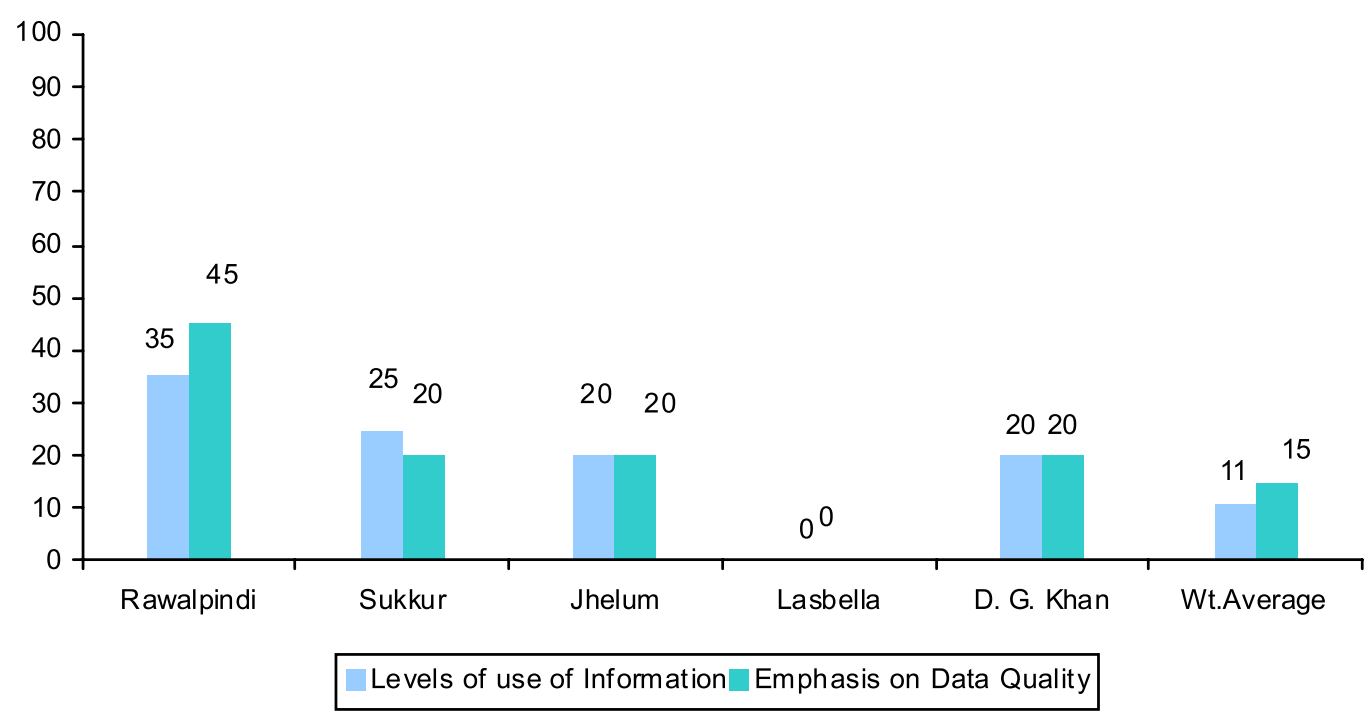

\section{Level of Feedback from District /Tehsil Office}

Feedback to the health facilities from higher authorities is an essential link in the process of use of information for performance improvement. It is a reflection of the culture of information use within the organization and the support provided by the higher authorities in promoting information use. The instructional manual on HMIS of Government of Pakistan, 2000 (Revised Edition) prescribes mechanisms for feedback. Districts are supposed to provide feedback in the form of monthly reports comparing performance of first level care facilities (FLCFs) and the yearly summary report. The leve 1 of feedback on data accuracy or facility performance based on HIS data from District or Tehsil office was assessed by us. The presence of a written do cument, e.g. letter or meeting minutes received within last 3 months from district or tehsil office was taken as the evidence of feedback to the health facility from the district or tehsil office.

Figure- 4 indicates that feedback is a weak area. Not a single facility in Sukkur, Lasbella and D. G. Khan received feedback from the district/tehsil office, while in Jhelum the level of feedback was 50 percent. This illustrates the lack of importance given to HISs. The PRISM questionnaire was finalized after a survey in Rawalpindi district, so the question of written feedback was not asked in the assessment of Rawalpindi. In the absence of feedback, data quality remains poor and data may not be used as such. Also, health facility staff remains unaware of 
decisions taken at the district level. It appears that there are no instructions from federal or provincial tiers that the minutes of the monthly meetings at the district office should be sent to facilities for taking necessary action. There might be verbal instructions provided by the district/tehsil health mangers to the facility staff, but usually that is not sufficient to stimulate any action at the facility level. A system of regular feedback is a weakness and an area that needs lots of attention.

\section{Figure 4: Feedback Received from District/Tehsil Health Managers}

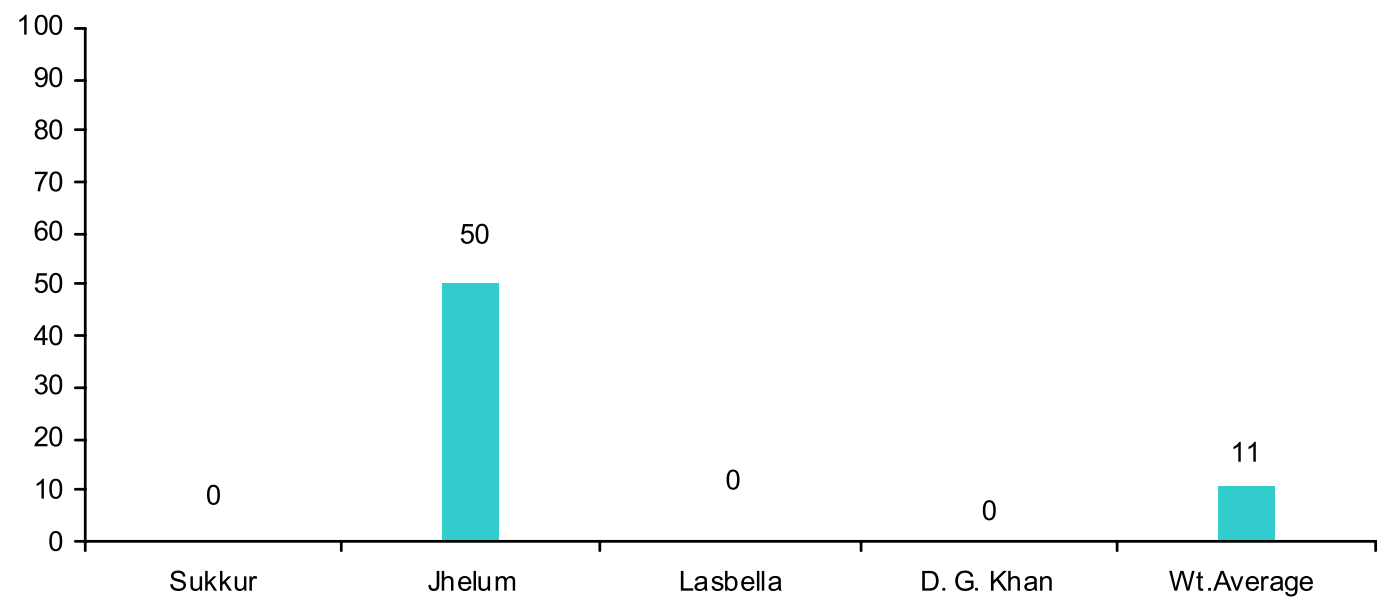

\section{Level of Data Completeness}

We evaluated data quality by its completeness. An incomplete report loses its value and definitely affects aggregate reports. This ultimately minimizes the usefulness of the data produced at the facility level. If data is incomplete, this is alsoindicativeoflackofmotivationorpropertrainingofdatareporting personnel on HMIS/HIS. The Instructional manual on HMIS of Government of Pakistan, 2000 (Revised Edition) clearly provides the following instructions for completing the monthly reports:

1. Fill in All items of the Report. Never leave blank a possible entry. If the number of the item is zero, fill in 0 .

2. For activities that are normally not performed in the health facility, the reporting section can be crossed out and overwritten by "Not Applicable"

Data completeness was assessed by examining the number of cells in the monthly reports that were left blank, i.e., neither filled with " 0 " nor crossed-out 
as "Not Applicable (N/A)". Since the facility staff was not asked to fill-in the cells for calculating percentages (\%) in the monthly HMIS report, these cells were excluded from the assessment. Reports with 80 percent or more of their cells filled were considered as adequately complete. Figure-5 below shows the level of more completeness of facility-based monthly reports, viz. Monthly HMIS reports, monthly LHW-MIS facility reports prepared by Lady Health Supervisor (LHS), monthly report prepared by LHW and monthly EPI reports from the facility.

Figure 5: Data Completeness of MIS Reports Submitted by the Health Facilities

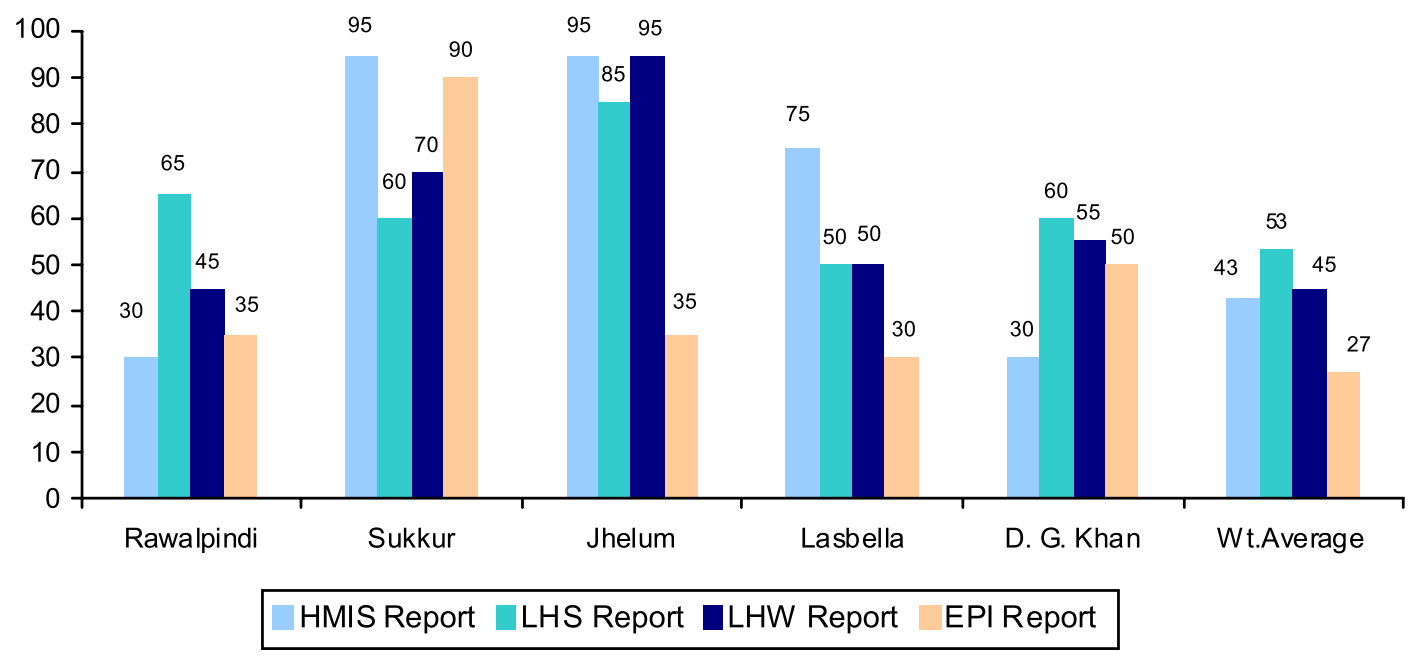

Assessment findings (Figure 5) show that overall average levels of the completeness of the monthly reports were 43 perent for HMIS, 53 percent for LHS reports, 45 percent for LHW reports and 27 percent for EPI reports, indicating that data completeness is a serious issue which needs to be properly addressed. District wise data completeness varies: it is below the desired level in the districts of Rawalpindi, Lasbella and DG Khan. In Sukkur and Jhelum the level of data completeness of HMIS reports is above 95 percent and that of other reports is also above the average levels for the five districts. 


\section{Level of Data Accuracy}

Data accuracy can be defined in two ways; whether the data recorded in the registers accurately represents the actual number of patients/clients provided the corresponding service; whether the data reported in the monthly reports is consistent with the data recorded on different registers. For the purpose of the assessment, data accuracy was assessed by cross-checking the data in the reports with that of the registers (records).

Initially during the assessment in Rawalpindi, the "Number of pregnant women newly registered" was selected to cross-check the level of data accuracy of HMIS reports. However, when the results were shared with the district managers, the complexity of record keeping using the mother health register and the nonavailability in many health facilities of Lady Health Visitors (LHVs) responsible for maintaining that register were pointed out. Based on that, a second data item "Number of female OPD patients aged 1-4 years" was randomly selected and added in the Assessment Tool.

Similarly, the data item "Number of post-natal cases visited within 24 hours" was randomly selected from the LHW/LHS monthly reports to assess the LHWMIS. During the exercise in Rawalpindi, it was found that the registers/ diary maintained by the LHWs do not have provision for clearly recording the number of post-natal cases visited within 24 hours. Usually, the visit information is recorded in the "Comments" column against the name of the pregnant women in the LHW's Diary. Others were just counting the number of deliveries occurring in the previous month and were also reporting them as postnatal cases visited within 24 hours. Nowhere in the records was the time of visit recorded. Nevertheless, the explanation that number of deliveries from the records can be assumed to be the same as the number of postnatal cases visited within 24 hours was accepted and the data in the records and reports were cross-matched accordingly.

During the assessments carried out in Sukkur, Jhelum, Lasbella and D. G. Khan the indicator for assessing LHW-MIS was replaced by two other randomly selected data items from the monthly reports. These were:

- Number of pregnant women provided iron tablets during the reporting month" and

- Number of new family planning clients 
There was no clear record in any of the LHW registers or diaries about the provision of iron tablets to specifically pregnant women. Rather the reporting is done assuming that all currently pregnant women have been provided iron tablets. In the "Treatment Register" the records of women (pregnant or nonpregnant/anemic) provided iron tablets is maintained, but there was no distinction between pregnant and non-pregnant women. This means that LHWs are supposed to report the number of pregnant women that were provided iron tablets but there is no provision in LHW's diary to record this indicator. Therefore, this data item was also dropped from the analysis; only the "Number of new Family Planning clients" was used for cross-checking data accuracy of LHW/LHS reports.

In the case of EPI register, the vaccinator/LHV maintains two registers: EPI Daily Register and EPI Permanent Register. The Daily Register is meant for recording daily activity while the Permanent Register is for longitudinal recording of the vaccination status of individual child or woman. Both the registers are supposed to be kept regularly updated. For data accuracy assessment the "Number of pregnant women receiving second tetanus toxoid (TT2)" shot from the EPI monthly report was considered.

Initially, it was decided to match the data from EPI permanent register, but it was soon found that this register is often not updated; rather the daily register is used for preparing the monthly report. Therefore, data in EPI reports were matched with the EPI Daily Register. Further, it was found that in the daily register or permanent register there is no provision to record whether the TT dose is given to a pregnant women or non-pregnant women. However, we were told that as a national policy, only pregnant women are given TT shots. Nonpregnant women were given TT shots only as part of special campaigns in some selected districts. Thus, the total figure of TT2 shots given in monthly report was compared with the EPI Daily Register. 
Figure 6: Data Accuracy at Health Facilities

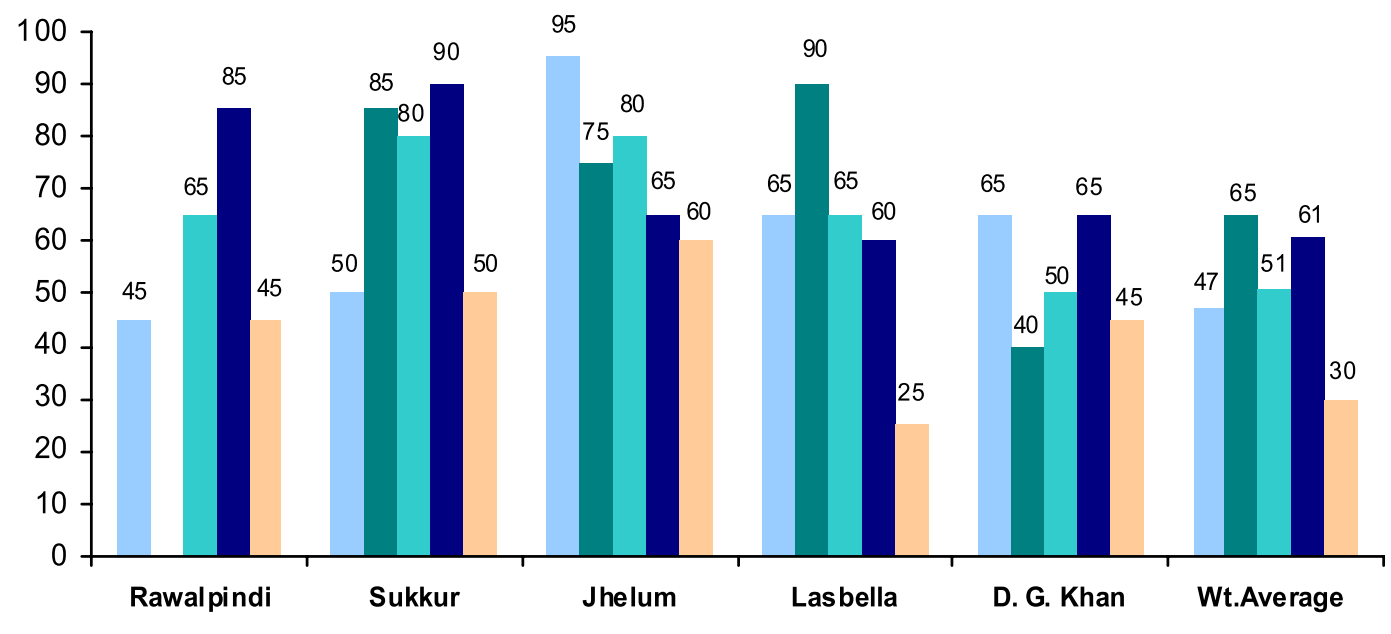

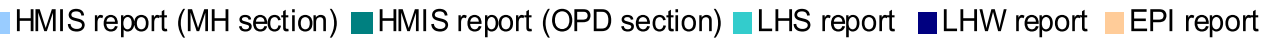

Figure- 6 provides the level of data accuracy of various routine HISs. In general, 80 percent data accuracy is considered as an acceptable level. Accuracy of HMIS data transferred from Mother Health Register was far below that level except in Jhelum. This substantiates the statement made by the district managers in Rawalpindi about the register as mentioned above. The level of accuracy of HMIS data from the OPD register was above 80 percent in Sukkur and Lasbella, but was not so in Jhelum and D.G. Khan. The levels of accuracy of data in LHW reports in Rawalpindi and Sukkur were above the 80 percent mark but the levels of data accuracy in LHS reports were 80 percent in Sukkur and Jhelum. It is interesting to note here that this discrepancy is reflected by the fact that the data in LHW reports did not match with that in the LHS report in many cases. The EPI data were not accurate in any district. The average level of data accuracy of the various HISs reports was: HMIS report-MH Section (47\%), HMIS ReportOPD Section (65\%), LHS report (51\%), LHW report (61\%) and EPI report (30\%). 


\section{Level of Training}

The training of Facility in-charges is a very important determinant of HIS data quality and the use for decision making. If the in-charge is better trained himself with HISs knowledge, he/she will be in a better position to guide the staff who fill and deal with data; his/her supervisory and monitoring role will be strengthened and he/she will have better skills for evidence-based decision making. Under the PAIMAN project, health facility in-charges were trained in HMIS in all of the 10 districts. We assessed the proportion of facility in-charges who have received training on HMIS, LHamW-MIS and EPI-MIS. Rawalpindi is not included in the analysis as during assessment in Rawalpindi, questions of HIS training were not asked from the facility in-charges; these were added later during the assessment carried out in the remaining districts.

Training of Facility in-charges on HMIS varied from 55 percent to 90 percent and the average level was 74 percent. Very few facility in-charges received the training on EPI: on average 23 percent facility in-charges with no one in Lasbella received training on EPI-MIS. The level of training of facility in-charges on LHWMIS also varied widely, between 0 percent in Lasbella to 75 percent in DG Khan with an average of 25 percent facility in-charges received training on LHW-MIS in the 4 districts.

\section{Figure 7: HIS Training of Facility In-charge}

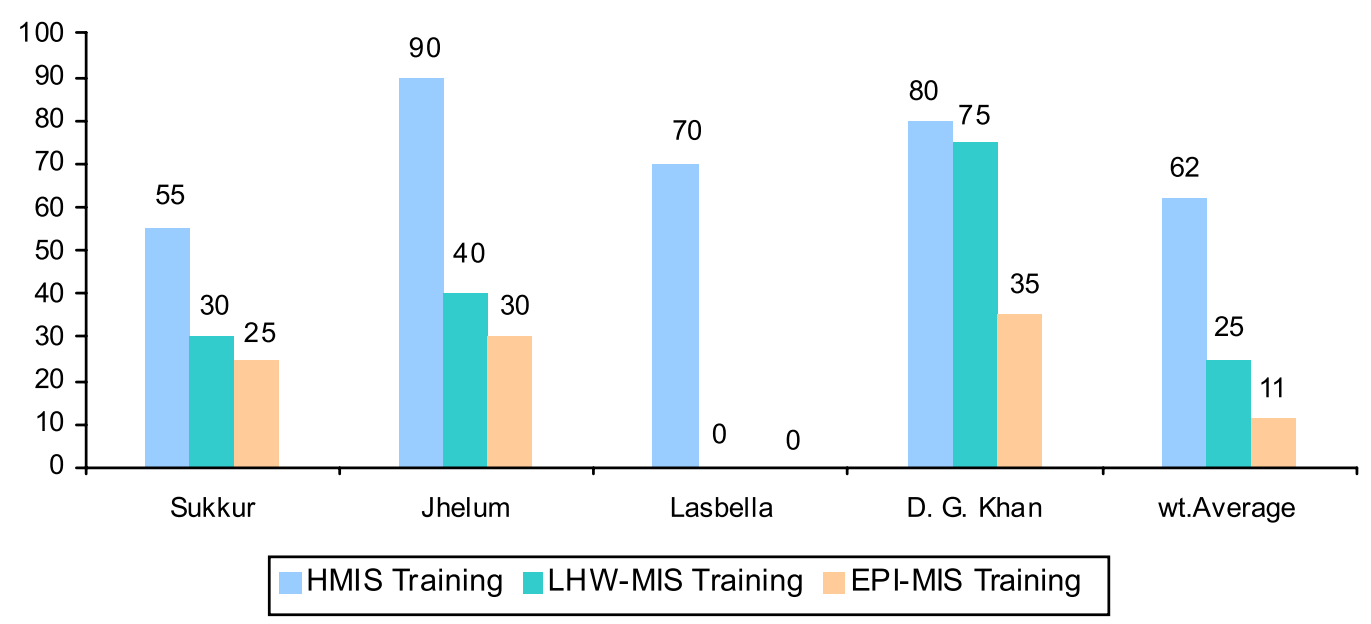




\section{Conclusions and Summary Findings}

The primary objective of facility assessment was to build the capacity of district health managers for effective monitoring and evaluation of programs using LQAS technique. This objective was achieved by conducting district level training on LQAS in which participants were given theoretical orientation followed by a facility based data collection. Data collected from the facilities was tabulated by the district health managers and results were discussed with the respective EDO (Health) and other district health managers.

We found the following situation of management information system (MIS)at the health facilities of the district: although the MISs are operative at health facilities, some considerable gaps regarding HMIS resources, quality, completeness and accuracy of data were identified. At most of the health facilities in all districts, HIS resources (reporting forms) were available in sufficient number. Generally there is a lack of feedback from the district/tehsil office to the health facilities regarding quality and use of data. Although the process of HMIS is usually discussed at monthly departmental meetings (EDO health office), written feedback in the form of letter etc. is not sent to the facilities. We discussed whether the data collected from the health facility each month is discussed among the facility staff for the use of HIS information. Overall only $11 \%$ health facilities internally discuss the monthly HIS data, with a similar situation across 5 districts. This is an area of potential improvement and quality of data could be better emphasized though discussions at the facility level.

The display of information at the health facilities through charts, tables or graphs was observed regarding total monthly patients, antenatal, natal and postnatal clients. A third of facilities across 5 districts display these information. The majority of facilities in district Jhelum and Rawalpindi display above mentioned information while a quarter in Sukkur and a third in D.G. Khan displayed the information. No information was displayed in district Lasbella. Some gaps were identified in completely filling the monthly reports of various HISs. Most of the LHS reports were complete, while slightly less than half of the LHW and facility HMIS reports were completed. Only a quarter of EPI reports were completely filled across five districts. An overwhelming majority of HMIS reports were completely filled in district Sukkur, Jhelum and Lasbella compared to less than a third properly filled HMIS reports in Rawalpindi and D.G. Khan Districts. Lady Health Supervisors and LHW reports were completely filled at majority of health facilities in 5 districts. Most of the health facilities in Sukkur and D.G. Khan had 
completely filled the EPI reports compared to around a third in Lasbella, Jhelum and Rawalpindi.

Data accuracy is the most important issue regarding HIS. This was better in HMIS reports followed by LHW reports, LHS reports and least in EPI reports across five districts. Among the two HMIS report sections (Maternal Health section and OPD section), a greater level of accuracy was found in the OPD section compared to $\mathrm{MH}$ (maternal health) section reported by LHV. Less than half of the facilities in District D. G. Khan showed accuracy of LHS and HMIS data. Otherwise across the districts data accuracy was found at majority of health facilities. The majority of health Facility in-charges (62\%) received HMIS training during last 3 years. Only a quarter of health facilities in-charges received LHWMIS training and only $11 \%$ received EPI-MIS training. It is apparent from those results that there are some potentially important areas in MIS which can be identified using LQAS assessments and addressed accordingly. 


\section{Recommendations}

The following recommendations are based on the findings of the HISs assessment carried out in the five Project districts. Basically, the recommendations focus on how to improve data quality (completeness and accuracy) and use of HIS information at facility and district levels.

\section{Recommendations for improving data completeness and accuracy:}

a. Existing written guidelines/instructions should be provided to the facility in-charges and staff. These guidelines, especially those relating to commonly mistaken record-keeping or reporting issues should be communicated verbally to the staff during the monthly meetings of the facility in-charges at EDOH Office and monthly meeting of facility staff at facility level. Special sessions should be arranged for staff on areas where data quality is the poor/unsatisfactory, e.g. Mother Health Register

b. District Managers and Facility in-charges should also be provided guidelines on how to assess data accuracy at the facility level using LQAS method. The district managers can apply this method during their regular supervisory visits. Based on the findings they could provide technical support to the staff for improving the data quality and use.

\section{Recommendations for improving use of information generated by routine HISs:}

a. As an initial step, the district managers should be sensitized on use of HIS data for evidence-based decision making. Similarly, the Nazims and DCOs should also be sensitized on the usefulness of the HIS data for performance monitoring and planning.

b. Facility in-charge should hold regular facility staff meetings at the health facilities where data accuracy and facility performance (including that of LHW program and EPI) are discussed.

c. The, EDOH during his monthly meetings and the DHMT during their meetings should review district/facility performance using the HIS data and take necessary decisions for performance improvement. The 
minutes of these meetings should be circulated in a timely manner to all the Facility in-charges for their information and necessary action.

d. The EDOH should circulate written instructions to the facility incharges to maintain minutes of all the meetings held at the health facility. A meeting minutes' format should be circulated to all the facilities for that purpose.

e. During their regular supervisory visits, district supervisors, should review these minutes and arrange necessary support to the facilities.

f. The EDOH/DOH should arrange the printing and supply of predesigned wall-chart formats for displaying monthly trends of important indicators. These should be decided through consultations catering directly to the district's information needs. Necessary directives should also be sent to the facility in-charges for maintaining updated charts in the health facilities. The district supervisors should check how well updated the wall-charts are during their regular supervisory visits.

3. DHDCs in the districts should work out a system of regular in-service training and refresher training of facility staff on various HISs.

4. At the national level there is a need to review the design of various HISs; e.g. there is a need to improve the record-keeping formats for properly recording information on data items such as, supply of iron tablets to pregnant women, postnatal visits etc. 


\section{Annex-I}

\section{Introduction to Prism Framework}

The ultimate objective of routine health information system is to produce quality information that is actively used for taking actions to improve health system's performance ${ }^{1}$. MEASURE Evaluation, together with John Snow, Inc, developed a conceptual framework, known as PRISM (Performance of Routine Information System Management), which defines that the performance of routine HIS is not measured by how much data it produces, but by the level of data quality and continuous use of information generated by the system ${ }^{2}$. In this regard, a technically sound HIS is important for generating quality data, but the overall success of HIS in terms of production of quality data and continuous use of information for management decision-making depends on factors far beyond the technical capabilities, such as:

- Organizational committed to a culture of using information

- Skills of the staff responsible for data collection

- Understanding, motivation and care about the importance data collection, reporting and analysis

- Support of the managers through training, supervision and resources

The PRISM framework acknowledges the broader context in which Routine HIS operates and, therefore, broadens the analysis of routine health information systems to include three key factors for success ${ }^{1}$ :

1. Behavioral determinants - knowledge, skills, attitudes, values, and motivation of the people who collect and use data

2. Technical determinants - Data collection processes, systems, forms, and methods.

3. Organizational/environmental determinants - Information culture, structure, resources, roles, and responsibilities of the health system and key contributors at each level.

The PRISM conceptual framework and Tools identify strengths and weaknesses in these areas, as well as correlations among the areas. This assessment aids in designing and prioritizing interventions to improve Routine HIS performance, which in turn improves the performance of the health system.

\footnotetext{
${ }^{1}$ PRISM Tools: Performance of Routine Information System Management - Four tools to improve the quality and use of routine health data. Data Demand and Information Use. Part Two: Strategies and Tools. MEASURE Evaluation
}

2 PRISM paper: Introducing an Analytical Framework for Understanding the Performance of the Routine Health Information System in Development Countries, September 2003, Dr. Theo Lippeveld, et al. 


\section{Annex. II \\ HMIS Record Keeping Registers /Chart}

1. OPD Register

2. Abstract Register

3. Child Health Register

4. Mother Health Register

5. Family Planning Register

6. TB Register

7. IDD Register

8. Laboratory Register

9. Daily EPI Register

10. Permanent Register

11. Population Chart of Catchments Area

12. Birth Register

13. Stock Register: Medicine/Supplies

14. Stock Register: Equipment/Furniture /Linen

15. Meeting Register

16. Daily Expense Register

17. Attendance Register

18. Log Book

19. Stock Register: Vaccine

* In addition to above there are a number of patient /client record cards. 


\section{JSI}

JSI Research \& Training Institute, Inc.

CA \# 36098 project is funded by the United States Agency for International Development and implemented by JSI Research \& Training Institute, inc.

in conjunction with Aga Khan University, Contech International, Greenstar Social Marketing, JHU-

CCP, PAVHNA, Population Council, Save the Children USA 